\title{
Radiation tolerance and mitigation strategies for FPGA:s in the ATLAS TileCal Demonstrator
}

\author{
H. Åkerstedt ${ }^{1 *}$, on behalf of the ATLAS Tile Calorimeter System \\ ${ }^{*}$ This is a collaborative work with the following main contributors: \\ K. Anderson ${ }^{2}$, C. Bohm ${ }^{1)}$, G. Drake ${ }^{3)}$, S. Muschter ${ }^{1)}$, M. Oreglia ${ }^{2)}$, F. Tang ${ }^{2}$ \\ ${ }^{1)}$ Fysikum, Stockholm University, Sweden \\ ${ }^{2}$ University of Chicago, USA \\ Argonne National Laboratory, USA
}

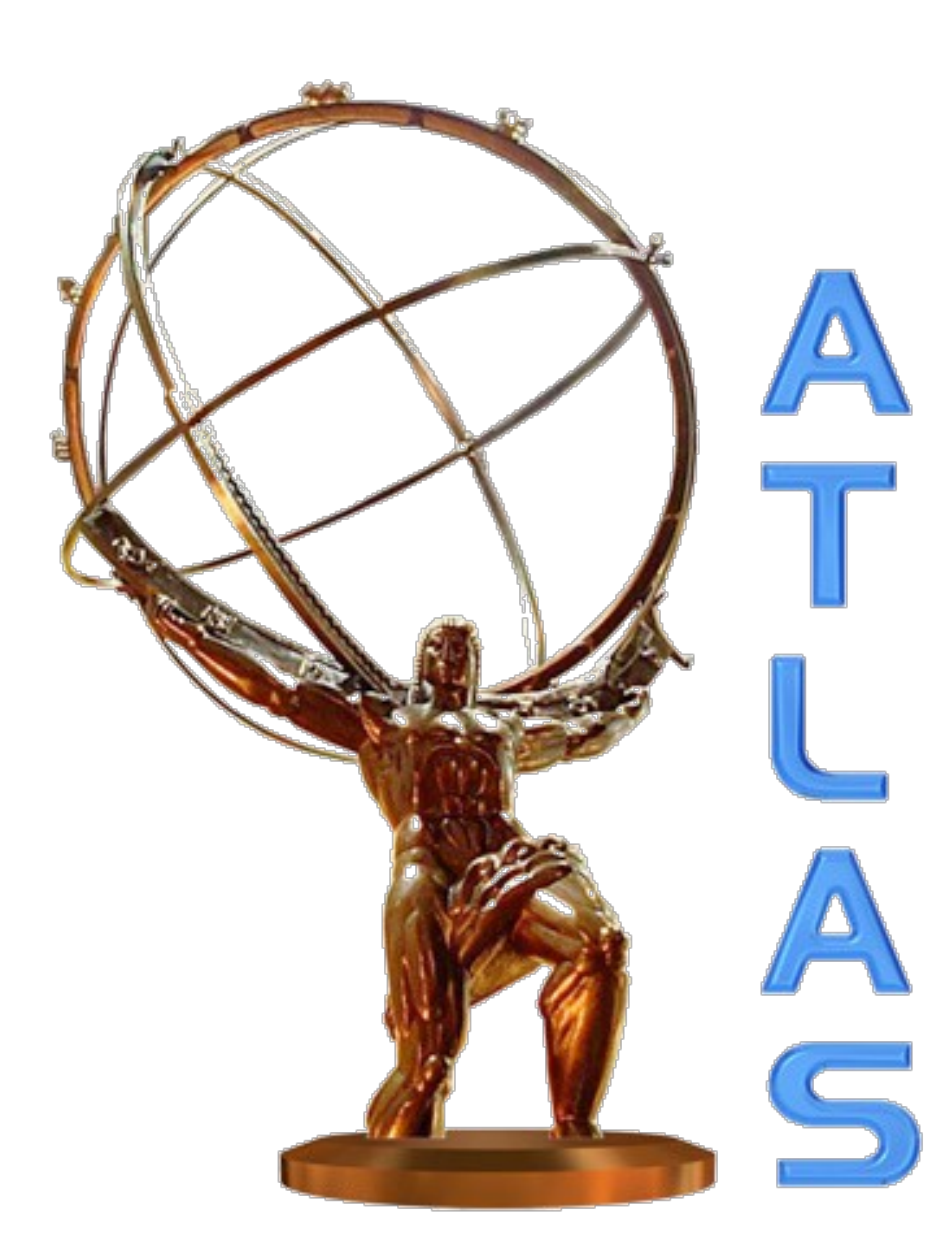

\section{Introduction}

The electronics of the hadron calorimeter of ATLAS, TileCal, need to be upgraded both to extend the detector lifetime and to able to cope with the higher radiation levels expected from the LHC luminosity upgrades.

-The current system transmits only merged analog tower sums to the Level-1 trigger and reads out detailed digital data for accepted events. In the upgraded system all data will be read out digitally.

-Detector interventions will become more problematic in the future as the detector parts will be activated by long time exposure at high radiation levels. To avoid excessive detector intervention we must design for highest reliability.

-Due to the rather moderate radiation levels expected at the place for our electronics we have chosen to base the design on KINTEX-7. We do not expect the ionizing radiation to be a problem due to the small feature sizes (28nm). However, SEUs will occur and must be mitigated
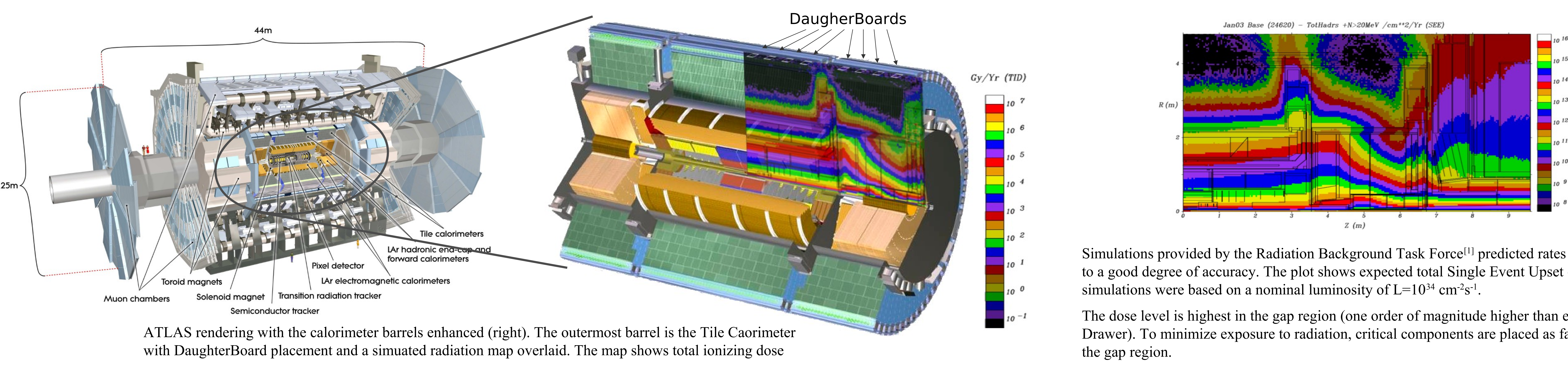

Simulations provided by the Radiation Background Task Force ${ }^{[l]}$ predicted rates for various detector parts to a good degree of accuracy. The plot shows expected total Single Event Upset (SEU) rate. These simulations were based on a nominal luminosity of $\mathrm{L}=10^{34} \mathrm{~cm}^{2} \mathrm{~s}$

The dose level is highest in the gap region (one order of magnitude higher than elsewhere in the Tile Drawer). To minimize exposure to radiation, critical components are placed as far away as possible from the gap region.

-To gain experience with the upgraded design we have developed the TileCal upgrade demonstrator, which is designed to evaluate the new design as well as operate transparently within the current system. We intend to install four such modules in ATLAS in mid 2014.

\section{Reliability Strategy}

To achieve necessary reliability the following steps were taken:

-Choose reliable radiation tolerant components that minimize loss at faliures -Avoid single point failure modes -Use redundancy to improve reliability -Use available FPGA error mitigation techniques for SEU:s

-When possible place equipment in least exposed areas

-Simplify extraction of electronics drawers to minimize radiation exposure

\section{Implementation of reliable readout system}

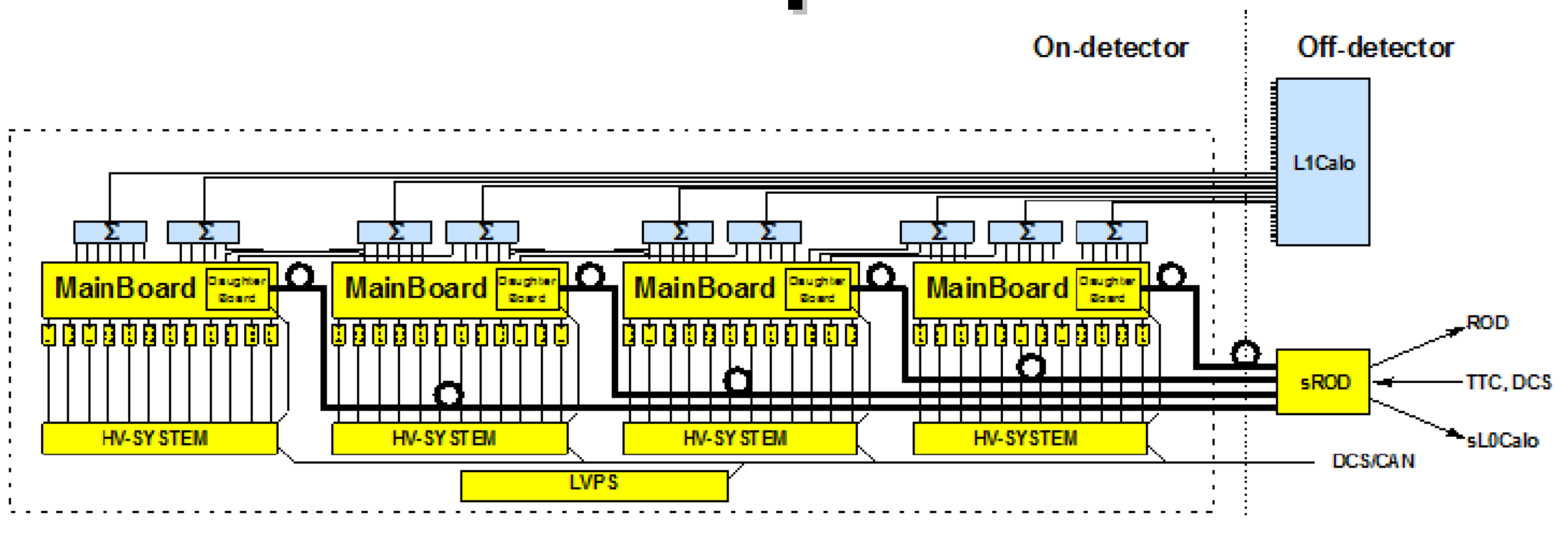

System wide:

Small independent units (half mini drawers) serving 6 channels each Redundant (duplicated) up and down going fiber links

Redundant LVPS -2 independent $10 \mathrm{v}$ bricks for each mini drawer

Local POL regulators

MainBoard, DaughterBoard and HVPS contain 2 separate but functionally identical parts - each part serves the same the same set of calorimeter cells but different PMTs (there are 2 PMTs/cell). If one side fails of data can still be read out.

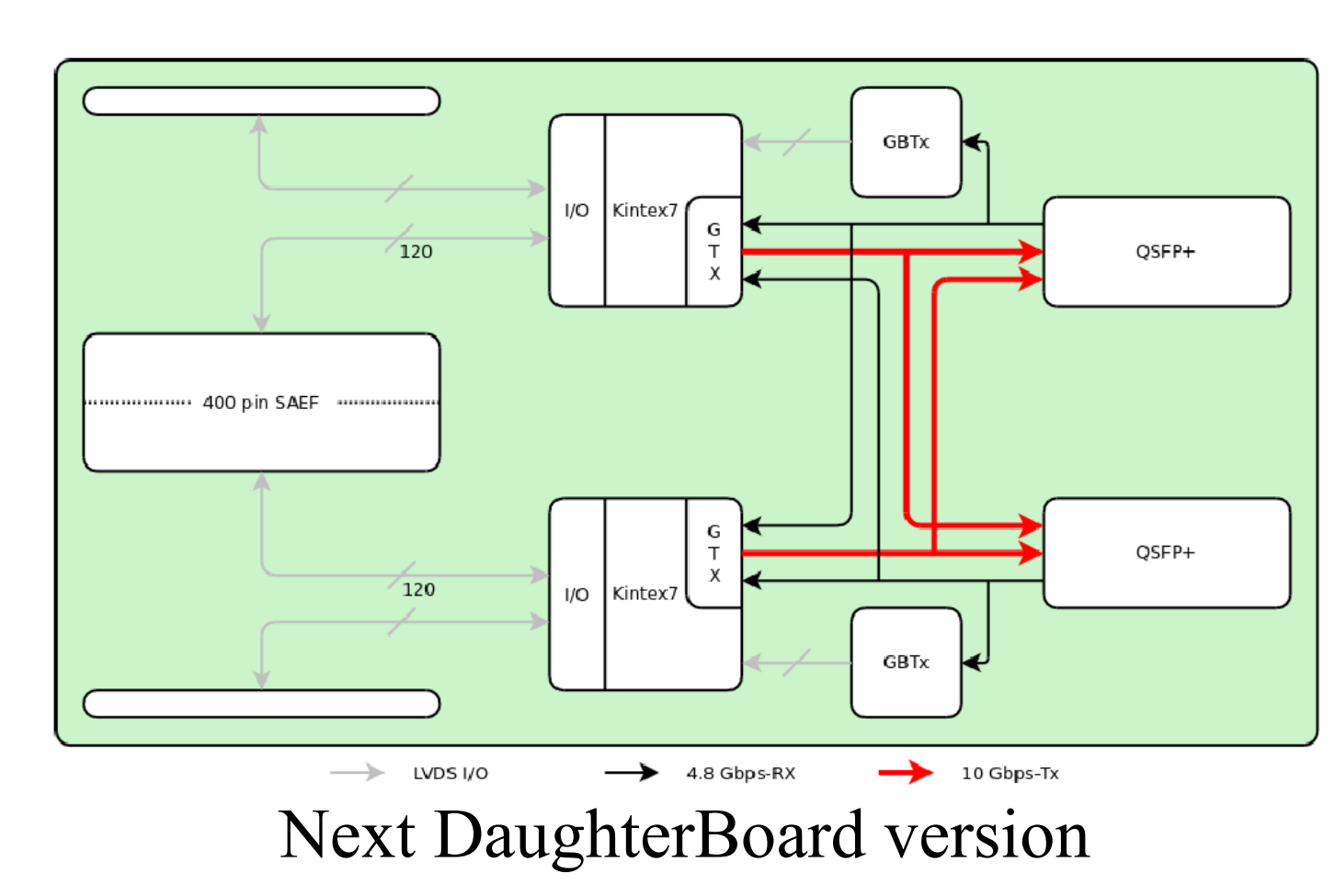

Board wide (DaughterBoard)

Symmetric parts with two Kintex-7 FPGAs

Kintex configured via link and rad hard protocol chip GBTX ${ }^{[2]}$

Duplicated rad tolerant Luxterra 10 Gbps QSFP modulators ${ }^{[3]}$

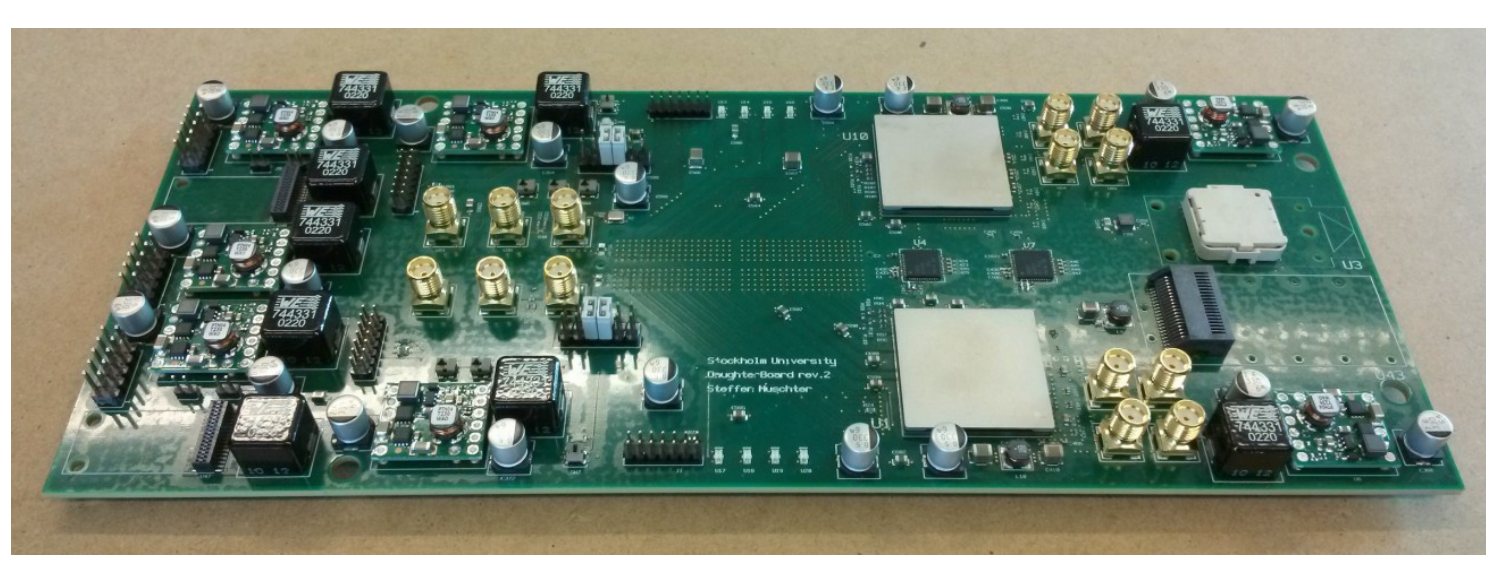

Present DaughterBoard prototype

\section{FPGA-specific reliability}

- Two JTAG chains with cross connections - one side can monitor and initiate reconfiguration of the other side

- FPGA configuration via JTAG over link and radiation hard GBTX chip

- Internal scrubbing of configuration memory repairs single errors and adjacent two bit errors

- Cross monitoring of scrubbing status via watchdog and serial interface

- If scrubbing is unsuccessful, it reports errors off-detector and to neighboring Kintex for reconfiguration

- Possibility to do partial reconfiguration by loading affected frames via an SPI interface from off-detector memory

- Four small Altera FPGAs on the MainBoard can be reconfigured via off-detector links

- Selective triple redundant logic

Based on the radiation level estimation above and tests made by other groups ${ }^{[4]}$, we expect an upset rate of:

- 3 upsets/day in the configuration memory

- 1 upset/day in BlockRAM

This is well within what the scrubbing can handle.

Uncorrectable multi bit upsets are expected to occur at a 10-100 times lower rate. Essential bits are expected to be around $10 \%$.

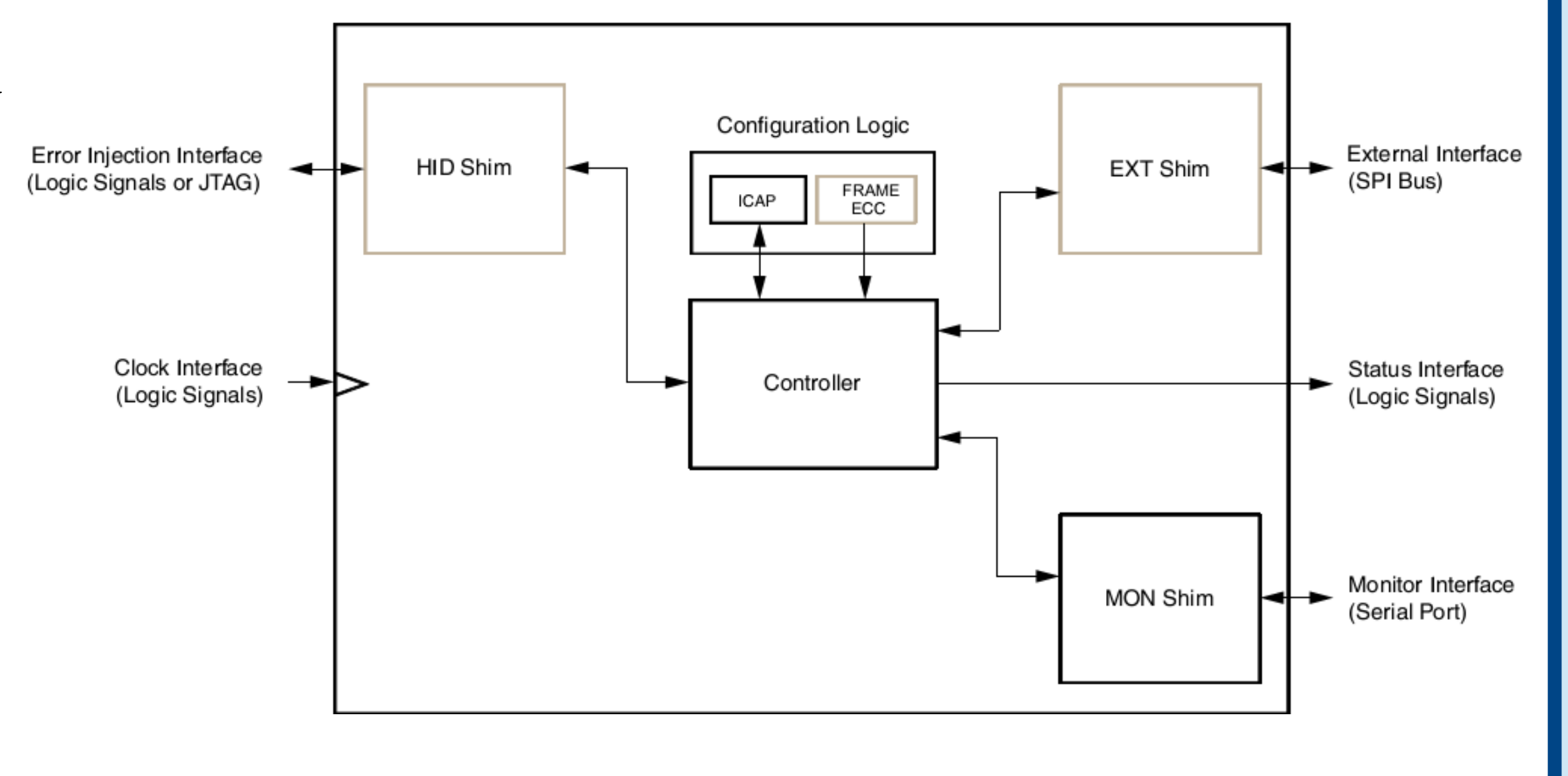

\section{Outlook}

Initial radiation testing will be conducted with a firmware that implements optical link loopback, configuration scrubbing, RAM and flip-flops. The tests will show whether our current mitigation strategy is sufficient or if more techniques are needed.

\section{References}

1. http://bosman.web.cern.ch

osman/Radiation maps.htm

3. mouser.com/new/molex/molex-qsfp-plus/

Ne "Soft Error Rate Estimation of the Kintex-7

FPGA within the ATLAS Liquid Argon (LAr) Calorimeter" 